Campos, Y.A.C.; Vianna, J.M.; Guimarães, M.P.; Souza, H.L.R.; Silva, G.P.; Domínguez, R.; Silva, S.F.; Novaes, J.S. y Reis, V.M. (2021). Prediction of the Anaerobic Threshold in the Leg Press $45^{\circ}$ Exercise. Revista Internacional de Medicina y Ciencias de la Actividad Física y el Deporte vol. 21 (81) pp. 83-97 Http://cdeporte.rediris.es/revista/revista81/artprediccion1211.htm

DOI: https://doi.org/10.15366/rimcafd2021.81.006

\title{
ORIGINAL
}

\section{PREDICCIÓN DEL UMBRAL ANAERÓBICO EN EL EJERCICIO PRENSA DE PIERNAS A 45 ${ }^{\circ}$}

\section{PREDICTION OF THE ANAEROBIC THRESHOLD IN THE LEG PRESS $45^{\circ}$ EXERCISE}

Campos, Y.A.C. ${ }^{1,2}$; Vianna, J.M. ${ }^{2}$; Guimarães, M.P. ${ }^{1,3}$; Souza, H.L.R. ${ }^{1,4}$; Silva, G.P. ${ }^{1 ;}$ Domínguez, R. ${ }^{5}$; Silva, S.F. ${ }^{1,6}$; Novaes, J.S. ${ }^{2,7}$ y Reis, V.M. ${ }^{8}$

1, 6 Grupo de Estudio y Investigación en Respuestas Neuromusculares, Universidad Federal de Lavras / Programa de Postgrado en Nutrición y Salud, Universidad de Lavras (Brasil) sandrofs@ufla.br

1,2 Grupo de Estudio y Investigación en Respuestas Neuromusculares, Universidad Federal de Lavras/Programa de Postgrado de la Facultad de Educación Física y Deportes, Universidad Federal de Juiz de Fora (Brasil) reiclauy@hotmail.com

2 Programa de Postgrado de la Facultad de Educación Física y Deportes, Universidad Federal de Juiz de Fora (Brasil) jeferson.vianna@ymail.com

2, 7 Programa de Postgrado de la Facultad de Educación Física y Deportes, Universidad Federal de Juiz de Fora/Universidad Federal del Rio de Janeiro (Brasil) jsnovaes@terra.com.br

1,3 Grupo de Estudio e Investigación en Respuestas Neuromusculares, Universidad Federal de Lavras/Facultad Presbiteriana GAMMON (Brasil) millerguimaraes@yahoo.com.br

${ }^{4}$ Grupo de Estudio e Investigación en Respuestas Neuromusculares, Universidad Federal de Lavras / Programa de Postgrado de la Facultad de Educación Física y Deportes, Universidad Federal de Juiz de Fora (Brasil) hlrsouza@gmail.com

5 Facultad de Ciencias de la Salud, Universidad Isabel I (España) raul dominguez herrera@hotmail.com

${ }^{8}$ Centro de Investigación en Ciencias del Deporte, Ciencias de la Salud y Desarrollo Humano, Universidad de Trás-os-Montes y Alto Douro (Portugal) victormachadoreis@gmail.com

Agradecimientos: Este trabajo recibió financiación de la Fundación Portuguesa para la Ciencia y la Tecnología - FCT (UID04045 / 2020)

Código UNESCO / UNESCO code: 2411 Fisiología del Ejercicio / Exercise Physiology

Clasificación Consejo de Europa / Council of Europe Classification: 6. Fisiología del Ejercicio / Exercise Physiology

Recibido 26 de enero de 2019 Received January 26, 2019

Aceptado 18 de abril de 2019 Accepted April 18, 2019 


\section{RESUMEN}

Objetivos: Identificar el umbral anaeróbico (UAnaer) a través de las concentraciones de lactato sanguíneo (UANAERDMÁx) y mediante la percepción subjetiva del esfuerzo (RPE) por la metodología Dmáx (URPEDMÁx), y evaluar la correlación entre estos métodos. Métodos: Dieciséis corredores varones participaron del estudio. Los participantes realizaron un test incremental progresivo en el ejercicio de prensa de piernas a $45^{\circ}$ registrando las concentraciones de lactato sanguíneo y RPE. Un test $T$-Student se realizó para comparar la intensidad de ejercicio en la que se encontró el UANAERDMÁx y URPEDMÁx y se aplicó un test Pearson para verificar la correlación y el coeficiente de correlación intraclase (ICC). Resultados: No se encontró diferencia significativa entre los métodos UANAERDMÁx y $U_{\text {RPEDMÁx }}(p<0,05)$. Además, se observó una correlación fuerte $(r=0,73)$ y un ICC alto $(0,822)$ entre ellos. Conclusiones: Para la muestra estudiada fue posible determinar el UAnaer a través de la cinética de la RPE.

PALABRAS CLAVE: fisiología del ejercicio, resistencia, umbral aeróbico, umbral anaeróbico, transición aeróbica-anaeróbica

\section{ABSTRACT}

Objective: Identify the anaerobic threshold (AnT) through blood lactate concentrations (LTDmax) and rating of perceived exertion (RPE) by the Dmax method (RPETDmax), and evaluate the correlation between these methods.

Methods: Sixteen male long-distance runners participated in the study. Participants performed a gradual incremental test in leg press $45^{\circ}$ exercise collecting blood lactate concentrations and perceived exertion. A student's Ttest was performed to compare the intensity of exercise in which the LTDmax and RPETDmax were found, and a Pearson test was applied to verify the correlation and intraclass correlation coefficient (ICC). Results: No significant difference was found between the LTDmax and RPETDmax $(p<0.05)$. In addition, a strong correlation $(r=0.73)$ and high ICC (0.822) were observed between them. Conclusions: For the sample studied, it was possible to determine the AnT through the kinetics of RPE.

KEYWORDS: exercise physiology, endurance, aerobic threshold, anaerobic threshold, aerobic-anaerobic transition.

\section{INTRODUCCIÓN}

El concepto de umbral anaeróbico (UAnaer) está siendo utilizado en los últimos cincuenta años tanto en el contexto clínico como deportivo (1), donde es considerado un importante indicador del rendimento en resistencia cardiorrespiratoria (2). Una de las formas de determinación del UAnaer es a través del análisis de las concentraciones de lactato sanguíneo (3), mediante el máximo estado estable de lactato (MLSS), intensidad de ejercicio asociada con la potencia crítica (4) y considerada por los fisiólogos de Ciencias del Deporte 
como el patrón de referencia en la valoración del rendimiento de la resistencia cardiorrespiratoria $(1,5,6)$. El MLSS se relaciona con la mayor intensidad de ejercicio en la que se mantienen estables las concentraciones de lactato en sangre, es decir, existe un equilibrio entre la tasa de producción y de aclaramiento de lactato (7), lo que permite mantener el esfuerzo durante un alto período de tiempo antes de que aparezca la fatiga (8). El principal inconveniente del MLSS es que la determinación requiere de varias sesiones de valoración en las que el deportista debe acudir al laboratorio (9). De este modo, los deportistas deben realizar pruebas sucesivas de 30 minutos de duración a intensidad constante con cargas situadas entre el umbral láctico y el consumo de oxígeno pico $\left(\mathrm{VO}_{2 \text { pico }}\right)$, identificándose al MLSS como la intensidad de ejercicio más alta en la que las concentraciones de lactato sanguíneo no aumenta más de $1 \mathrm{mmol} \cdot \mathrm{I}^{-}$ ${ }^{1}$ entre los 10 y 30 minutos (10). Con objeto de reducir el número de sesiones, así como la complejidad en la determinación del MLSS, se han propuesto protocolos de determinación más simples para estimar éste UAnaer a partir de un test incremental máximo (11).

Tradicionalmente, el UAnaer ha sido determinado en modalidades de ejercicio con una naturaleza cíclica mediante tests incrementales $(6,7,11)$. Sin embargo, recientemente, algunas investigaciones han comenzado a determinar éste parámetro en ejercicios empleados para el entrenamiento de la fuerza $(3,12$, 13). En esta última modalidad de ejercicio, al relativizar esta intensidad como porcentaje de una repetición máxima (\% 1RM) durante pruebas incrementales en ejercicios empleados para el entrenamiento de la fuerza (prensa de piernas con $45^{\circ}$ de inclinación), se ha determinado el UAnaer en intensidades próximas al $30 \%$ en sujetos de edad avanzada $(14,15), 31 \%$ en sujetos con diabetes tipo 2 (16) y entre el 32,3 y $36,6 \%$ en sujetos con experiencia en el entrenamiento de fuerza $(17,18)$. Recientemente, en una investigación se ha propuesto que el entrenamiento de fuerza realizado a una intensidad de UAnaer puede ser un entrenamiento efectivo para provocar mejoras en parámetros tanto de fuerza como de resistencia cardiorrespiratoria (19), siendo adecuada para la programación del entrenamiento de fuerza en individuos que buscan mejorar su salud y calidad de vida, así como en deportistas que buscan mejorar su rendimiento atlético (20). Sin embargo, ninguna investigación hasta la fecha ha determinado el UAnaer en ejercicios empleados para el entrenamiento de la fuerza en deportistas de resistencia cardiorrespiratoria, dificultando la programación de este tipo entrenamiento en esta población.

Debido a que la determinación del lactato sanguíneo es una prueba invasiva y costosa, algunas investigaciones emplearon la monitorización de la percepción subjetiva del esfuerzo (RPE) con objeto de predecir el UAnaer a través de la misma durante pruebas incrementales tanto en ejercicios cíclicos empleados para el desarrollo de la resistencia cardiorrespiratoria (21-23) como en ejercicios empleados para el desarrollo de la fuerza (24). En este contexto, la RPE se convertiría en una alternativa más económica que el lactato sanguíneo (23), disminuyendo los costes y reduciendo la complejidad de la determinación (24, 25). Además, la escala OMNI ya ha sido utilizada en ejercicio empleados para el entrenamiento de fuerza, donde ha demostrado ser una herramienta válida en la predicción del gasto energético durante el esfuerzo (26). 
Borg (27) propuso la RPE como un método válido para la determinación del UAnaer, ya que, ésta representa la integración de señales centrales y periféricas de varios sistemas, siendo un indicador tanto de la frecuencia cardíaca, lactato sanguíneo o ventilación durante el ejercicio (28). De este modo, algunas investigaciones han encontrado correlaciones fuertes entre el UAnaer, mediado a través de las concentraciones de lactato sanguíneo (21-24) y a través de la RPE (URPE). Sobre todo cuanto el UAnaer y el URPE se determinaron mediante la metodología Dmáx, propuesta por Cheng et al. (29), el $U_{R P E}$ podría ser un buen predictor del UAnaer $(21,22,24)$, pudiendo ser un instrumento no invasivo, de bajo coste y complejidad, de identificación del UAnaer en corredores durante la realización de ejercicios empleados para el entrenamiento de la fuerza.

\section{OBJETIVO}

Los objetivos del presente trabajo de investigación han sido identificar el UAnaer a través de las concentraciones de lactato sanguíneo mediante la metodología Dmáx (UANAERDMÁx) y la RPE a través de la metodología Dmáx (URPEDMÁx). El segundo objetivo de la investigación fue evaluar la concordancia y correlación entre UANAERDMÁx y URPEDMÁx durante un test incremental en el ejercicio de prensa de piernas a $45^{\circ}$ en corredores entrenados. Nuestras dos hipótesis de investigación son: i) es posible determinar UA NAERDMÁx y U RPEDMÁx durante un test incremental en prensa de piernas en corredores de resistencia; ii) existe una concordancia y correlación alta entre ambas determinaciones y la escala de determinación de RPE OMNI-RES.

\section{MATERIAL Y MÉTODO}

\section{Sujetos}

Dieciséis corredores aficionados de resistencia del sexo masculino (edad: 27,28 $\pm 2,54$ años; porcentaje de grasa corporal: $9,98 \pm 3,52 \%$; estatura: $1,78 \pm 0,54$ m; masa corporal: 74,22 $\pm 5,58 \mathrm{~kg}$ ) con experiencia en competiciones de $5000 \mathrm{y}$ 10000 metros (5000 m: 21 minutos y 28,7 segundos \pm 1 min y 24 segundos; experiencia de entrenamiento: $3,26 \pm 0,89$ años y con sus marcas aproximadamente en promedio al $60 \%$ del record brasileño de los $5000 \mathrm{~m}$ en carretera) voluntariamente participaron en el presente estudio. Los criterios de inclusión para la participación en el estudio fueron los siguientes: a) ser mayor de edad; b) no presentar ninguna patología ósea, muscular y cardiovascular; c) participar regularmente en carreras de 5000 y $10000 \mathrm{~m}$ (al menos 6 competiciones anuales); d) tener experiencia en el entrenamiento de fuerza y, particularmente, con el ejercicio de prensa de piernas a $45^{\circ}$; e) no haber ingerido ningún suplemento nutricional ni fármaco en los 3 meses previos al inicio de la investigación; f) no ser considerado deportista de élite. Previo a la realización de la investigación, en conformidad con la Declaración de Helsinki, el estudio fue aprobado por el comité de ética local (CAAE: 53675416.3.0000.5148), firmando todos los participantes un consentimiento informado tras asistir a una sesión 
informativa en la que se informó del procedimiento y se resolvieron todas las dudas que los participantes tenían.

\section{Diseño experimental}

Los sujetos realizaron 6 sesiones de valoración en el laboratorio en la misma franja horaria ( $\pm 0,5$ horas) con las mismas condiciones ambientales $\left(20^{\circ} \mathrm{C}\right.$ y una humedad relativa del $60 \%$ ) separadas entre sí por un período de 48 horas. En la $1^{\circ}$ sesión se tomaron medidas antropométricas de los participantes y se les informó del procedimiento que se llevaría a cabo en las siguientes sesiones, al tiempo que se administró el consentimiento informado. En la $2^{\circ}$ y $3^{\circ}$ sesión los sujetos realizaron un test de valoración de $1 \mathrm{RM}$ en el ejercicio de prensa de piernas a $45^{\circ}$. En la $4^{\circ}$ sesión, los sujetos realizaron una sesión de familiarización con la escala OMNI-RES en el ejercicio de prensa de piernas a $45^{\circ}$. En la $5^{\circ}$ y $6^{\circ}$ sesión, los sujetos realizaron un test incremental progresivo en el ejercicio de prensa de piernas a $45^{\circ}$ registrando las concentraciones de lactato sanguíneo o de RPE en la finalización de cada escalón con distintas cargas de trabajo, siendo la secuencia de las dos sesiones aleatorizadas en todos los sujetos.

\section{Valoración antropométrica}

Para realizar la caracterización de la muestra se realizó una toma de datos antropométricos. De este modo, la estatura y la masa corporal se valoraron mediante una balanza con estadiómetro (110 FF, Welmy ${ }^{\circledR}$, Santa Bárbara d'Oeste, Brasil) y la estimación del porcentaje de grasa corporal se realizó mediante bioimpedancia eléctrica (Quantum BIA-II, RJL Systems $®$, Clinton Township, USA), utilizando electrodos de tipo tetrapolar (Bio Tetronic, Sanny®, São Bernardo do Campo, Brasil).

\section{Test de 1 RM}

La determinación de 1RM se realizó siguiendo las directrices de la National Strength and Conditioning Association (30). El test dio inicio tras un calentamiento específico realizando series con cargas más livianas. De este modo, el test de inició con una primera serie con una carga estimada en torno al $50 \%$ de 1RM, a partir de la carga que empleaban los sujetos para realizar regularmente en sus entrenamientos series con una carga de 8 a 10 repeticiones máximas. Tras una recuperación de 2 a 4 minutos de recuperación pasiva, hasta que los sujetos tenían una percepción completa de la recuperación, se fueron imponiendo cargas progresivas con incrementos de un $10 \%$ con respecto a la anterior carga de trabajo. Cuando los sujetos llegaron al fallo, se redujo la carga un $5 \%$. El 1 RM se estableció como la carga más alta que los sujetos desplazaron manteniendo una técnica de ejecución correcta.

\section{Familiarización con escala OMNI-RES}

Durante el proceso de familiarización, la escala OMNI-RES fue presentada a los participantes al mismo tiempo que se les explicó que la misma era una escala 
que mediante descriptores visuales y numéricos para clasificar el esfuerzo percibido durante la realización del entrenamiento de fuerza. A continuación, de acuerdo con las recomendaciones de Robertson et al., (31), los participantes leyeron una definición de la misma en conjunto. La escala fue utilizada a través de un procedimiento visual-cognitivo (32), siendo el objetivo principal que el sujeto estableciese una relación cognitiva entre la intensidad del esfuerzo con los descriptores visuales y numéricos de la escala. Los sujetos fueron instruidos para que memorizasen de menor a mayor el esfuerzo experimentado a medida que levantaban las cargas para ayudar a establecer un vínculo visual-cognitivo. Además, se mostró a los sujetos para que señalasen con el dedo su percepción del esfuerzo sobre la escala y no a responder verbalmente cuando la RPE fuese solicitada por los investigadores.

\section{Test incremental progresivo en prensa de piernas}

Durante la realización del test incremental progresivo en el ejercicio de prensa de piernas a $45^{\circ}$ (Physicus $®$, Auriflama, Brasil), los sujetos realizaron series de 1 minuto de trabajo en el que realizaban 20 repeticiones a un ritmo controlado de 20 repeticiones por minuto (1 segundo durante la fase concéntrica y 2 segundos durante la fase excéntrica) impuesta externamente mediante un metrónomo digital (DM90, Seiko $\Re$, Tokyo, Japan) $(33,34)$. Las cargas empleadas durante el test fueron del 10, 20, 25, 30, 35 y 40\% de 1 RM. A partir del $40 \%$ de $1 \mathrm{RM}$, los incrementos de la carga fueron de una magnitud del $10 \%$ de $1 \mathrm{RM}(50 \%, 60 \%$ de $1 \mathrm{RM} . .$.$) , dándose por finalizado el test cuando los sujetos$ no eran capaces de realizar 20 repeticiones durante un estadio de trabajo al ritmo impuesto $(33,34)$. El período de recuperación entre cada serie de trabajo fue de 2 minutos, en los cuales, se realizó una toma de lactado sanguíneo y la RPE. Durante toda la sesión, los sujetos permanecieron sentados en la máquina con el tronco con una inclinación horizontal de $45^{\circ}$ y una flexión de $90^{\circ}$ de rodillas (15). Durante la realización del ejercicio, se realizó una extensión de rodilla y cadera para volver a la posición inicial de flexión (15). El movimiento fue controlado a través de un goniómetro digital (GN360, Miotec® Equipamentos Biomédicos Ltda, Porto Alegre, Brasil).

\section{Medición del lactato sanguíneo}

Inmediatamente, después de la finalización de cada carga de trabajo, un investigador tomó una muestra de sangre utilizando lancetas (Accu-Chek SafeT-Pro Uno, Roche ${ }^{\circledR}$, Hawthorne, USA) y guantes desechables (Cremer®, Blumenau, Brasil), a partir de una punción realizada en el lóbulo de la oreja. Tras desechar la primera gota de sangre, se recolectaron $25 \mu \mathrm{L}$ de sangre arterial.

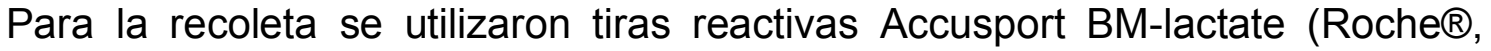
Hawthorne, USA) y un analizador portátil de lactato (Accusport, Boehringer Mannheim - Roche®, Hawthorne, USA), previamente validado y fiabilizado (35). Antes del inicio de cada sesión de valoración, el analizador de lactato fue calibrado a partir de distintas soluciones patrón de concentraciones de lactato conocidas $\left(2,4,8\right.$ e $\left.10 \mathrm{mmol}^{-l^{-1}}\right)$. 


\section{Medición de la percepción subjetiva del esfuerzo}

Para la medición de la RPE durante el test incremental progresivo en prensa de piernas se empleó la escala OMNI-RES (31), con sus correspondientes descriptores visuales y numéricos que varían desde "extremamente fácil" (0) a "extremamente difícil" (10). A los 30 segundos de haber finalizado cada serie de trabajo durante el test incremental los sujetos manifestaron su RPE en base a la escala OMNI-RES. Durante toda la prueba los sujetos tuvieron a la vista la escala, si bien, en las repeticiones finales de cada serie se les indicó a los participantes que "pensasen sobre su percepción de esfuerzo durante el ejercicio".

\section{Determinación de UA NAERDMÁx y U RPEDMÁx mediante la metodología Dmáx}

El método Dmáx (29) se aplicó a partir de un ajuste polinomial de $2^{\circ}$ orden entre la cinética de las concentraciones de lactato sanguíneo (UANAERDMÁX) y de la RPE (URPEDMÁx $)$ en función de la intensidad del ejercicio, seguido de un ajuste lineal, derivándose una ecuación para cada ajuste. Posteriormente, los valores " $x$ " de las ecuaciones fueron sustituidos por las intensidades absolutas de cada carga empleada durante el test incremental progresivo. Los valores obtenidos fueron sustituidos en la ecuación (lineal - polinomial), y Dmáx se consideró como la mayor diferencia observada (Figura 1 y Figura 2).

\section{Determ in ación del U A NAERMÁX}

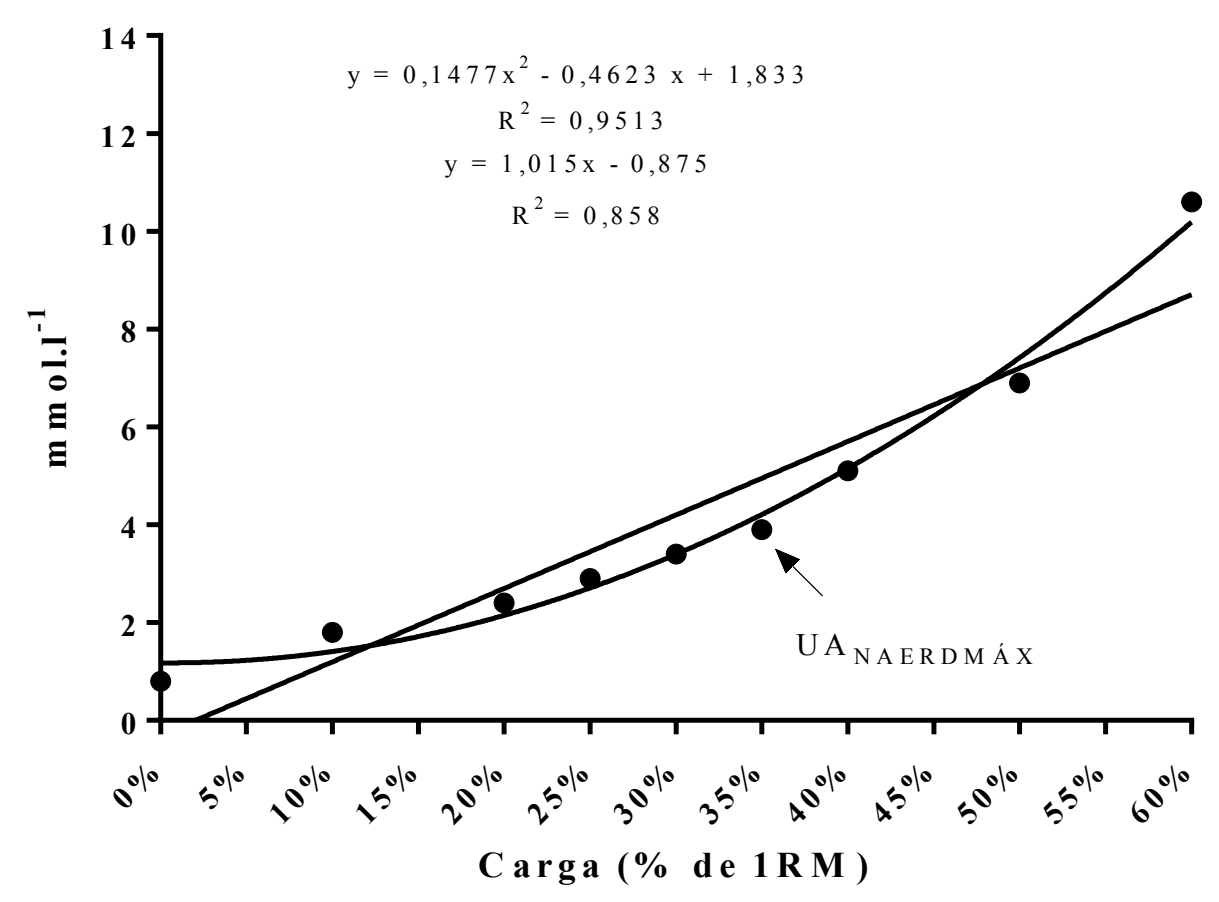

Figura 1. Determinación de UANAERDMÁx durante el test incremental progresivo en prensa de piernas $45^{\circ}$ 


\section{Determ in ación del U RPEMÁX}

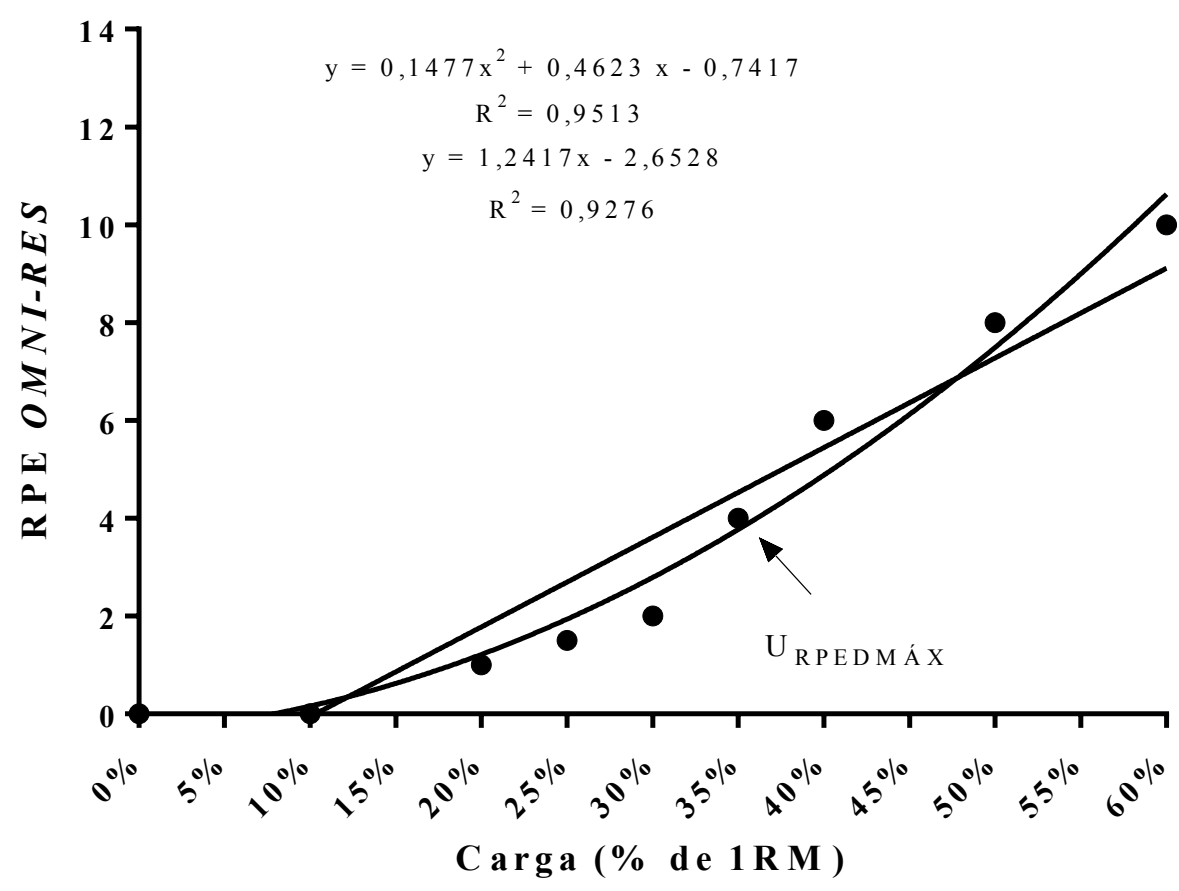

Figura 2. Determinación de URPEDMÁx durante el test incremental progresivo en prensa de piernas $45^{\circ}$

\section{ESTADÍSTICA}

Para verificar la normalidad y homogeneidad de varianzas se aplicaron los tests de Shapiro Wilk y Levene. Al cumplirse los presupuestos de normalidad e igualdad de varianzas en todas las variables, se aplicó un test T-Student para muestras relacionadas para realizar el análisis de la intensidad de ejercicio (\% de $1 R M$ ) en la que se encontró el UANAERDMÁx y URPEDMÁx durante el test incremental progresivo en el ejercicio de prensa de piernas $45^{\circ}$. El cálculo del tamaño del efecto (ES) se realizó en base al test de Cohen (d), a partir de la siguiente fórmula: $d=$ (media del grupo 1 - media del grupo 2) / desviación estándar. Para comprobar la correlación entre los métodos UANAERDMÁX y URPEDMÁx se aplicó un test Pearson. La interpretación de la correlación se realizó en base a la clasificación propuesta por Hinkle et al. (36): 0 - 0,30 despreciables; $0,30-0,50$ baja; $0,50-0,70$ moderada; $0,70-0,90$ fuerte y $0,90-1,00$ muy fuerte. Para comprobar el índice de concordancia entre UANAERDMÁX Y URPEDMÁX, se calculó el coeficiente de correlación intraclase $(\mathrm{CCl})$ Adicionalmente, para el análisis de concordancia entre UANAERDMÁX y URPEDMÁ, se realizó un análisis gráfico Bland-Altman (37). El valor de significación estadística asumida fue de $p<0,05$. Para el análisis estadístico se empleó el software SPSS (20.0, IBM, Armonk, USA). 


\section{RESULTADOS}

$\mathrm{El} \mathrm{CCl}$ entre el test y retest de 1RM fue de 0,962 (excelente). Al analizar la carga relativa (\% de 1RM) entre UANAERDMÁX Y URPEDMÁX no se obsevaron diferencias estadísticamente significativas ( $p=0,074, \mathrm{ES}=0,035$ : pequeño), al tiempo que se observó una correlación fuerte $(r=0,73)$ y un ICC alto $(0,822)$ (véase Tabla 1).

Tabla 1. Datos correspondientes a la comparación entre UANAERDMÁX y URPEDMÁx durante la realización del test incremental progresivo en el ejercicio de prensa de piernas $45^{\circ}$

\begin{tabular}{|c|c|c|c|c|c|}
\hline $\begin{array}{c}\text { Carga } \\
\text { Relativa } \\
\text { (\% de 1RM) }\end{array}$ & $\begin{array}{c}\text { Carga } \\
\text { Relativa } \\
\text { (\% de 1RM) }\end{array}$ & \multirow[t]{2}{*}{$\begin{array}{l}\text { Significación } \\
(p)\end{array}$} & \multirow[t]{2}{*}{$\begin{array}{c}\text { Correlación } \\
\qquad(r)\end{array}$} & \multirow[t]{2}{*}{$\begin{array}{l}\text { Significación } \\
\text { de la } \\
\text { Correlación } \\
\quad(p)\end{array}$} & \multirow[t]{2}{*}{$\mathrm{CCl}$} \\
\hline UANAERDMÁX & URPEDMÁX & & & & \\
\hline $38,3 \pm 2,9$ & $37,3 \pm 2,8$ & $p=0,074$ & $r=0,73^{*}$ & $p=0,001^{*}$ & 0,822 \\
\hline
\end{tabular}

* Diferencias estadística significativas $(p<0,05)$

En cuanto a las concentraciones de lactato en UANAERDMÁx éstas fueron de 5,05 $\pm 1,26 \mathrm{mmol} \cdot \cdot^{-1}$, mientras que los valores de RPE en U $\mathrm{RPEDMÁx}$ fueron de $5,37 \pm$ 1,36 puntos. En el análisis visual de concordancia mediante el gráfico de BlandAltman (Figura 3), se observa concordancia entre UA NAERDMÁx y URPEDMÁx durante el test incremental progresivo en prensa de piernas, estando el $100 \%$ de los datos dentro del intervalo de confianza $\pm 1,96$.

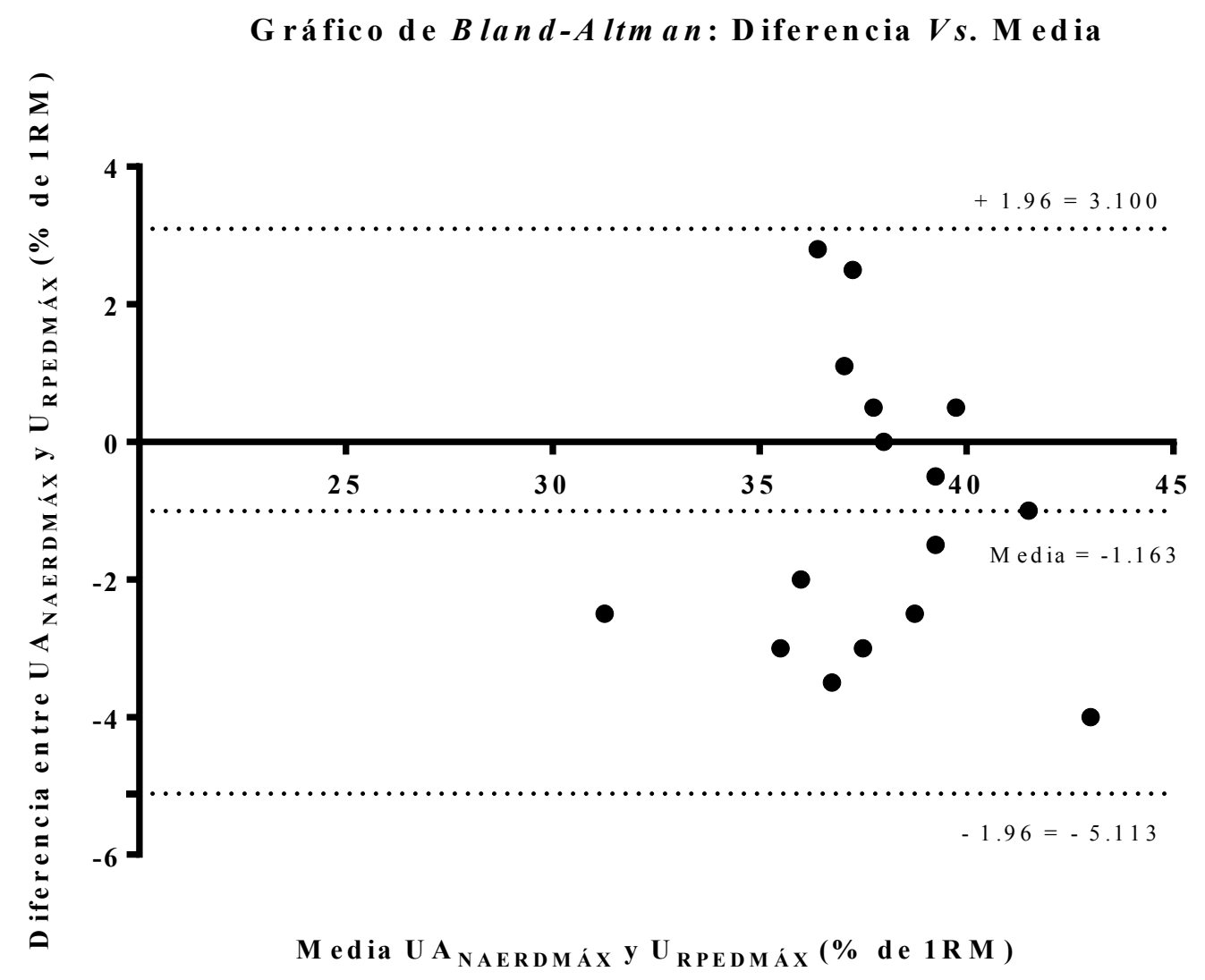

Figura 3. Concordancia entre UANAERdMÁx y URPEDMÁx como \% de 1RM. 


\section{DISCUSIÓN}

Los objetivos de nuestra investigación fueron identificar UAnaer mediante UA $A_{\text {NAERDMAX }}$ y URPEDMAX durante la realización del test incremental progresivo en el ejercicio de prensa de piernas $45^{\circ}$, así como analizar la correlación y la concordancia entre ambos parámetros. Nuestras hipótesis iniciales fueron confirmadas, de este modo, fue posible determinar el UAnaer a través de UANAERDMAX Y URPEDMÁx, existiendo una fuerte correlación y concordancia ente ambas metodologías de determinación del UAnaer.

Los mecanismos que permiten la determinación del UANAERDMÁx a partir de las concentraciones de lactato en sangre pueden ser explicadas por mecanismos hemodinámicos y fisiológicos (33). En este sentido, numerosas investigaciones han informado de una oclusión de los capilares sanguíneos de la musculatura activa (15) a intensidades de ejercicio superiores al $30 \%$ de 1 RM $(14-17,33,34)$. La oclusión provoca un bloqueo de los capilares (38) reduciendo significativamente el flujo sanguíneo (15) y, consecuentemente, la biodisponibilidad de oxígeno para la musculatura activa (15), induciendo a un incremento de la contribución glucolítica al metabolismo energético $(33,34)$. El aumento de la intensidad del ejercicio que provoca una mayor oclusión y, por tanto, una mayor contribución glucolítica (aumentando el reclutamiento de las unidades motoras glucolítica o tipo II en detrimento de las oxidativas o de tipo I) (15), estimula la actividad de la enzima lactato deshidrogenasa (39) dando lugar a un aumento de las concentraciones de lactato en sangre de tipo exponencial a medida que aumenta la intensidad del esfuerzo (15). De este modo, una reducción de la biodisponibilidad de oxígeno como consecuencia de la oclusión vascular, proporciona un reclutamiento de las unidades motoras con mayor actividad glucolítica que permite la determinación de $\operatorname{UAnaer}(33,34)$. Recientemente, en una revisión sistemática se ha concluido que UAnaer puede ser identificada en ejercicios empleados para el entrenamiento de fuerza en intensidades que oscilan entre el 27 y el $36 \%$ de 1 RM (20), sin embargo, en la presente investigación se ha comprobado que UANAERDMÁx tiene lugar a una intensidad ligeramente superior ( $38,3 \%$ de $1 \mathrm{RM})$. Una posible explicación en los valores superiores encontrados en nuestra investigación puede tener origen en los sujetos que conformaron la muestra. De este modo, Messonnier et al., (40) observaron que en deportistas de resistencia cardiorrespiratoria presentan una mayor capacidad de aclaramiento de lactato durante el ejercicio, por lo que, esa mayor capacidad de aclaramiento de lactato podría afectar a la cinética del lactato sanguíneo durante la realización de tests incrementales progresivos en ejercicios empleados para el entrenamiento de fuerza, dando lugar a la aparición de UAnaer a intensidades superiores en corredores de resistencia cardiorrespiratoria.

La popularización de las escalas de RPE para la determinación del UAnaer han despertado el interés de la comunidad científica con objeto de evaluar y/o validar las mismas con otros parámetros fisiológicos de referencia como las concentraciones de lactato sanguíneo (21-24) y la ventilación (41). Durante el esfuerzo, los aumentos de la RPE están relacionados con 
alteraciones en la homeostasis entre las que se encuentran la acidosis metabólica, el aumento del consumo de oxígeno o de la frecuencia cardíaca (28, $42,43)$. De este modo, la RPE durante el esfuerzo está mediada por alteraciones metabólicas y periféricas que tienen lugar a nivel periférico durante la contracción muscular y que serían percibidas por las terminaciones de los nervios aferentes III y IV, transmitiendo la información sensitiva directamente al sistema nervioso central (SNC) (44), estimulando las áreas sensoriales del cerebro. Por tanto, la RPE durante el esfuerzo podría estar influenciada por un feedback sensorial (45) englobando a todo el conjunto de sensaciones psicofisiológicas que serían las encargadas de modular éstas durante el esfuerzo. Entre las alteraciones de tipo metabólico que en la literatura se ha informado que podrían actuar como vías de señalización en el SNC, afectando a la RPE durante el esfuerzo, se encuentran las concentraciones de lactato sanguíneo (46). De este modo, en ejercicios empleados para el desarrollo de la resistencia cardiorrespiratoria se ha encontrado concordancia y una correlación fuerte entre UANAERDMÁx y URPEDMÁx (21-23). Así, en una investigación similar a la nuestra, Arsa et al. (24) al evaluar a sujetos entrenados en fuerza, encontraron concordancia y una correlación

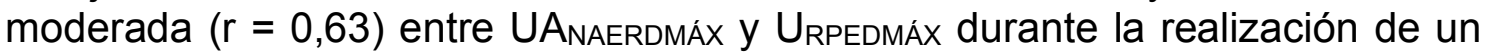
test incremental progresivo en el ejercicio de prensa horizontal. A diferencia de nuestra investigación, en la anterior investigación, Arsa et al. (24) utilizaron una escala de Borg (6-20). Sin embargo, nuestra investigación es la primera que ha empleado la OMNI-RES, escala validada específicamente para los ejercicios empleados en el entrenamiento de fuerza (31), para la determinación del $U_{\text {RPEDMÁx }}$ en ejercicios empleados para el entrenamiento de fuerza. Así, tanto la fuerte correlación $(r=0,73, p=0,074)$ como concordancia $($ ICC $=0,82)$ y los gráficos Bland-Altman (Figura 3), muestran una fuerte asociación entre UANAERDMÁx y URPEDMÁx como identificadores del UAnaer durante la realización de ejercicios empleados para el entrenamiento de fuerza. De este modo, nuestros resultados están alineados con otras investigaciones que han observado una fuerte correlación y concordancia entre UANAERDMÁx y URPEDMÁx en ejercicios empleados para el entrenamiento de la resistencia cardiorrespiratoria como sería la carrera en tapiz rodante $(21,23)$ o cicloergómetro (22).

A pesar de algunas diferencias acerca de los mecanismos responsables de la modulación de la RPE durante el esfuerzo (47), los resultados de nuestra investigación sugieren que ésta puede ser empleada en la determinación del UAnaer en los ejercicios empleados para el entrenamiento de la fuerza, siendo una herramienta económica y altamente aplicable en la monitorización y programación de la intensidad del ejercicio $(24,25)$, en relación con la utilización de las concentraciones de lactato sanguíneo (21-24) y de los parámetros ventilatorios (41), al exigir éstos equipamientos más sofisticados (23) que dificultan su utilización durante el entrenamiento y la competición de los deportistas (41). Adicionalmente, a nivel práctico, la realización de programas de entrenamiento de fuerza a intensidad de URPEDMÁx puede ser apropiada para deportistas de resistencia cardiorrespiratoria, ya que, se ha comprobado que a una intensidad de UAnaer se producen respuestas cardiorrespiratorias y mecánicas que lo proponen como una excelente oportunidad de desarrollar simultáneamente la resistencia cardiorrespiratoria y la fuerza muscular $(19,20)$. 


\section{CONCLUSIONES}

Los resultados demuestran que para la muestra estudiada fue posible determinar el UAnaer a través de la cinética de la RPE durante un test incremental progresivo en un ejercicio empleado para el entrenamiento de fuerza. De ese modo, nuestros resultados demostraron concordancia y correlación fuerte entre los métodos de determinación UANAERDMÁX y URPEDMÁX. Sobre todo, por el hecho de que la cinética de la escala OMNI-RES se asemeja mucho a la de las concentraciones de lactato sanguíneo durante el test progresivo en el ejercicio prensa de piernas a $45^{\circ}$. Sin embargo, recomendamos más estudios comprobar si la escala OMNI-RES puede ser recomendada para la valoración y prescripción de la intensidad del esfuerzo en el entrenamiento de fuerza para otras poblaciones.

\section{REFERENCIAS BIBLIOGRÁFICAS}

1. Messias LHD, Polisel EEC, Manchado-Gobatto FB. Advances of the reverse lactate threshold test: Non-invasive proposal based on heart rate and effect of previous cycling experience. PloS one. 2018;13(3):1-20. https://doi.org/10.1371/journal.pone.0194313

2. Billat $\mathrm{V}$, Sirvent $\mathrm{P}$, Koralszrein J, Mercier J. The concept of maximal lactate steady state: a bridge between biochemistry, physiology and sports science. Sport Med. 2003;33(6):407-26. https://doi.org/10.2165/00007256-200333060$\underline{00003}$

3. Maté-Muñoz JL, Domínguez R, Lougedo JH, Garnacho-Castaño MV. The lactate and ventilatory thresholds in resistance training. Clin Physiol Func Imaging. 2017;37(5):518-24. https://doi.org/10.1111/cpf.12327

4. Smith CG, Jones AM. The relationship between critical velocity, maximal lactate steady-state velocity and lactate turnpoint velocity in runners. Eur J Appl Physiol. 2001;85(1-2):19-26. https://doi.org/10.1007/s004210100384

5. Beneke R, von Duvillard SP. Determination of maximal lactate steady state response in selected sports events. Med Sci Sports Exerc. 1996;28(2):241-6. https://doi.org/10.1097/00005768-199602000-00013

6. Llodio I, Gorostiaga E, Garcia-Tabar I, Granados C, Sánchez-Medina L. Estimation of the maximal lactate steady state in endurance runners. Int J Sports Med. 2016;37(07):539-46. https://doi.org/0.1055/s-0042-102653

7. Wahl P, Zwingmann L, Manunzio C, Wolf J, Bloch W. Higher Accuracy of the Lactate Minimum Test Compared to Established Threshold Concepts to Determine Maximal Lactate Steady State in Running. Int J Sports Med. 2018; 39(7):541-48. https://doi.org/10.1055/s-0044-102131

8. Bosquet $L$, Léger $L$, Legros $P$. Methods to determine aerobic endurance. Sport Med. 2002;32(11):675-700. https://doi.org/10.2165/00007256-200232110$\underline{00002}$

9. Heck H, Mader A, Hess G, Mücke S, Müller R, Hollmann W. Justification of the 4-mmol/l lactate threshold. Int J Sports Med. 1985;6(03):117-30. https://doi.org/10.1055/s-2008-1025824 
10. Beneke R. Methodological aspects of maximal lactate steady state implications for performance testing. Eur J Appl Physiol. 2003;89(1):95-9. https://doi.org/10.1007/s00421-002-0783-1

11. Garcia-Tabar I, Gorostiaga EM. A "Blood Relationship" between the Overlooked Minimum Lactate Equivalent and Maximal Lactate Steady State in Trained Runners. Back to the Old Days? Front Physiol. 2018;13(3):1-13. https://doi.org/10.3389/fphys.2018.01034. eCollection 2018.

12. Garnacho-Castaño MV, Domínguez R, Ruiz-Solano P, Maté-Muñoz JL. Acute physiological and mechanical responses during resistance exercise at the lactate threshold intensity. J Strength Cond Res. 2015;29(10):2867-73. https://doi.org/10.1519/JSC.0000000000000956

13. Maté-Muñoz JL, Domínguez R, Barba M, Monroy AJ, Rodríguez B, RuizSolano $\mathrm{P}$, et al. Cardiorespiratory and metabolic responses to loaded half squat exercise executed at an intensity corresponding to the lactate threshold. J Sports Sci Med. 2015;14(3):648.

14. Simões R, Castello-Simões V, Mendes R, Archiza B, Santos D, Machado $\mathrm{H}$, et al. Lactate and heart rate variability threshold during resistance exercise in the young and elderly. Int J Sports Med. 2013;34(11):991-6. https://doi.org/10.1055/s-0033-1337946

15. Simões RP, Mendes RG, Castello V, Machado HG, Almeida LB, Baldissera V, et al. Heart-rate variability and blood-lactate threshold interaction during progressive resistance exercise in healthy older men. J Strength Cond Res. 2010;24(5):1313-20. https://doi.org/10.1519/JSC.0b013e3181d2c0fe

16. Moreira SR, Arsa G, Oliveira HB, Lima LC, Campbell CS, Simões HG. Methods to identify the lactate and glucose thresholds during resistance exercise for individuals with type 2 diabetes. J Strength Cond Res. 2008;22(4):1108-15. https://doi.org/10.1519/JSC.0b013e31816eb47c

17. Oliveira JC, Baldissera V, Simões HG, Aguiar AP, Azevedo PHSM, Poian PAFO, et al. Identificação do limiar de lactato e limiar glicêmico em exercícios resistidos. Rev Bras Med Esporte. 2006;12(6):333-8. http://dx.doi.org/10.1590/S1517-86922006000600007

18. Rocha RM, Bomfim DL, Nascimento TBR, Moreira SR, Simões HG. A Variação do método de incremento de cargas não altera a determinação do limiar de lactato em exercício resistido. Rev Bras Med Esporte. 2010;16(4):282-5. http://dx.doi.org/10.1590/S1517-86922010000400010

19. Domínguez R, Garnacho-Castaño M, San Juan A, Pérez-Ruiz M, GarcíaFernández $\mathrm{P}$, Veiga-Herreros $\mathrm{P}$, et al. Cardiorespiratory responses at the intensity of the threshold. Comparative study between half squat and cycloergometer. Rev Int Med Cienc Act Fís Deporte 2018;18(71): 507-20. https://doi.org/10.15366/rimcafd2018.71.007

20. Domínguez R, Maté-Muñoz J, Serra-Paya N, Garnacho-Castaño M. Lactate Threshold as a Measure of Aerobic Metabolism in Resistance Exercise. Int J Sports Med. 2018;39(3):163-72. https://doi.org/10.1055/s-0043-122740

21. Fabre N, Mourot L, Zerbini L, Pellegrini B, Bortolan L, Schena F. A novel approach for lactate threshold assessment based on rating of perceived exertion. Int J Sports Physiol Perform. 2013;8(3):263-70. https://doi.org/10.1123/ijspp.8.3.263

22. Ferreira GA, Osiecki R, Lima-Silva AE, de Angelis-Pereira MC, De-Oliveira FR. Effect of a reduced- $\mathrm{CHO}$ diet on the rate of perceived exertion curve during 
an incremental test. Int $J$ Sport Nutr Exerc Metab. 2014;24(5):532-42. https://doi.org/10.1123/ijsnem.2013-0248

23. Irving BA, Rutkowski J, Brock DW, Davis CK, Barrett EJ, Gaesser GA, et al. Comparison of Borg-and OMNI-RPE as markers of the blood lactate response to exercise. Med Sci Sports Exerc. 2006;38(7):1348-52. https://doi.org/10.1123/10.1249/01.mss.0000227322.61964.d2

24. Arsa G, Cambri LT, Silva Fd, Pardono E, Serra AJ, Leite GDS, et al. Anaerobic threshold from $P E$ in resistance exercise by mathematical models. Rev Bras Med Esporte. 2016;22(2):113-7. https://doi.org/10.1590/1517869220162202149738

25. Scherr J, Wolfarth B, Christle JW, Pressler A, Wagenpfeil S, Halle M. Associations between Borg's rating of perceived exertion and physiological measures of exercise intensity. Eur $J$ Appl Physiol. 2013;113(1):147-55. https://doi.org/10.1007/s00421-012-2421-x

26. Vianna J, Reis V, Saavedra F, Damasceno V, Silva S, Goss F. Can Energy Cost During Low-Intensity Resistance Exercise be Predicted by the OMNI-RES Scale? J Hum Kinet. 2011;29(Special Issue):75-82. https://doi.org/10.2478/v10078-011-0062-5

27. Borg G. Borg's perceived exertion and pain scales: Human kinetics; 1998.

28. Eston R. Use of ratings of perceived exertion in sports. Int J Sports Physiol Perform. 2012;7(2):175-82. https://doi.org/10.1123/ijspp.7.2.175

29. Cheng B, Kuipers H, Snyder A, Keizer H, Jeukendrup A, Hesselink M. A new approach for the determination of ventilatory and lactate thresholds. Int $\mathrm{J}$ Sports Med. 1992;13(07):518-22. https://doi.org/10.1055/s-2007-1021309

30. Baechle TR, Earle RW. Essentials of strength training and conditioning: Human kinetics; 2008.

31. Robertson RJ, Goss FL, Rutkowski J, Lenz B, Dixon C, Timmer J, et al. Concurrent validation of the OMNI perceived exertion scale for resistance exercise. Med Sci Sports Exerc. 2003;35(2):333-41. https://doi.org/10.1249/01.MSS.0000048831.15016.2A

32. Robertson RJ, Goss FL, Boer NF, Peoples JA, Foreman AJ, Dabayebeh IM, et al. Children's OMNI scale of perceived exertion: mixed gender and race validation. Med Sci Sports Exerc. 2000;32(2):452-8. https://doi.org/10.1097/00005768-200002000-00029

33. De Sousa N, Magosso R, Pereira G, Souza M, Vieira A, Marine D, et al. Acute cardiorespiratory and metabolic responses during resistance exercise in the lactate threshold intensity. Int J Sports Med. 2012;33(2):108-13. https://doi.org/10.1055/s-0031-1286315

34. De Sousa NMF, Magosso RF, Pereira GB, Leite RD, Arakelian VM, Montagnolli AN, et al. The measurement of lactate threshold in resistance exercise: a comparison of methods. Clin Physiol Funct Imaging. 2011;31(5):37681. https://doi.org/10.1111/j.1475-097X.2011.01027.x

35. Bishop D. Evaluation of the Accusport@ lactate analyser. Int J Sports Med. 2001;22(7):525-30. https://doi.org/10.1055/s-2001-17611

36. Hinkle DE, Wiersma W, Jurs SG. Applied statistics for the behavioral sciences. 2003.

37. Bland JM, Altman D. Statistical methods for assessing agreement between two methods of clinical measurement. The Lancet. 1986;327(8476):307-10. 
38. Williams MA, Haskell WL, Ades PA, Amsterdam EA, Bittner V, Franklin $\mathrm{BA}$, et al. Resistance exercise in individuals with and without cardiovascular disease: 2007 update a scientific statement from the american heart association council on clinical cardiology and council on nutrition, physical activity, and metabolism. Circulation. 2007;116(5):572-84. https://doi.org/0.1161/CIRCULATIONAHA.107.185214

39. Kohn T, Essén-Gustavsson B, Myburgh K. Specific muscle adaptations in type II fibers after high-intensity interval training of well-trained runners. Scand J Med Sci Sports. 2011;21(6):765-72. https://doi.org/0.1111/j.16000838.2010.01136.x

40. Messonnier LA, Emhoff C-AW, Fattor JA, Horning MA, Carlson TJ, Brooks GA. Lactate kinetics at the lactate threshold in trained and untrained men. J Appl Physiol.

2013;114(11):1593-602.

https://doi.org/10.1152/japplphysiol.00043.2013

41. Monnier-Benoit P, Groslambert A, Rouillon J-D. Determination of the ventilatory threshold with affective valence and perceived exertion in trained cyclists: a preliminary study. J Strength Cond Res. 2009;23(6):1752-7. https://doi.org/10.1519/JSC.0b013e3181b74dc1

42. Borg GA. Psychophysical bases of perceived exertion. Med Sci Sports Exerc. 1982;14(5):377-81.

43. Robertson RJ, Falkel JE, Drash AL, Swank AM, Metz KF, Spungen SA, et al. Effect of blood $\mathrm{pH}$ on peripheral and central signals of perceived exertion. Med Sci Sports Exerc. 1986;18(1):114-22.

44. Pageaux B, Gaveau J. Studies using pharmacological blockade of muscle afferents provide new insights into the neurophysiology of perceived exertion. $J$ Physiol. 2016;594(18):5049-51. https://doi.org/10.1113/JP272585

45. Peñailillo L, Mackay K, Abbiss CR. Rating of Perceived Exertion During Concentric and Eccentric Cycling: Are We Measuring Effort or Exertion? Int J Sports Physiol Perform. 2018;13(4):517-23. https://doi.org/10.10.1123/ijspp.2017-0171

46. Hampson DB, Gibson ASC, Lambert MI, Noakes TD. The influence of sensory cues on the perception of exertion during exercise and central regulation of exercise performance. Sports Med. 2001;31(13):935-52. https://doi.org/10.2165/00007256-200131130-00004

47. Abbiss CR, Peiffer JJ, Meeusen R, Skorski S. Role of ratings of perceived exertion during self-paced exercise: what are we actually measuring? Sports Med. 2015;45(9):1235-43. https://doi.org/0.1007/s40279-015-0344-5

Número de citas totales / Total reference number: 47 (100\%) Número de citas propias de la revista / Journal's own references: $1(2,12 \%)$

Rev.int.med.cienc.act.fís.deporte - vol. 21 - número 81 - ISSN: 1577-0354 